\title{
Percutaneous closure of large VSD using a home-made fenestrated atrial septal occluder in 18-year-old with pulmonary hypertension
}

\author{
Hacer Kamali ${ }^{1}$, Turkay Saritas ${ }^{1,3^{*}}$, Abdullah Erdem ${ }^{1}$, Celal Akdeniz ${ }^{2}$ and Volkan Tuzcu ${ }^{2}$
}

\begin{abstract}
Background: Hemodynamically significant muscular ventricular septal defects in children after the infantile period are a rare occurrence and ideal for transcatheter closure. In cases of severe concomitant pulmonary hypertension, it may be necessary to fenestrate the device. In this report, we present an 18-year old patient with a large midtrabecular ventricular septal defect and severe pulmonary hypertension that underwent percutaneous closure of the defect with a home-made fenestrated atrial septal occluder.

Case presentation: An 18-year-old female patient referred to us with complaints of dyspnea (NYHA score of 2-3). Physical examination revealed an apical rumble and a harsh second heart sound. Echocardiographic examination revealed a large mid-trabecular ventricular septal defect with bidirectional shunt and the widest diameter measuring $22 \mathrm{~mm}$ on 2D echocardiography. Left and right heart cavities were enlarged. Before and after the vasoreactivity test performed during cardiac catheterization, average aortic pressure was $65 \rightarrow 86 \mathrm{mmHg}$, average pulmonary artery pressure: $58 \rightarrow 73 \mathrm{mmHg}$, Qp/Qs: $1.6 \rightarrow 3.2$, PVR: $4.6 \rightarrow 4.3 \mathrm{Wood} / \mathrm{U} / \mathrm{m}^{2}$ and PVR/SVR: $0.5 \rightarrow 0.2$. On left-ventricular angiocardiogram, the largest end-diastolic defect diameter was $21 \mathrm{~mm}$. The closure procedure was performed with transthoracic echocardiographic guidance, using a $24 \mathrm{~mm}$ Cera septal occluder and a $14 \mathrm{~F}$ sheath dilator to make a 4.5-5 $\mathrm{mm}$ opening. Measured immediately after the procedure and during cardiac catheterization one month later, average aortic pressure was $75 \rightarrow 75 \mathrm{mmHg}$, average pulmonary artery pressure: $66 \rightarrow 30 \mathrm{mmHg}$, Qp/Qs $1.5 \rightarrow 1.4$, PVR: $4.4 \rightarrow 2.9$ Wood/U/ $\mathrm{m}^{2}$ and PVR/SVR: $0.4 \rightarrow 0.2$. Transthoracic echocardiographic examination performed 24 hours after the procedure showed a max $35-40 \mathrm{mmHg}$ gradient between the left and right ventricles through the fenestration. After the procedure, we observed sporadic early ventricular systoles and a nodal rhythm disorder that started after approximately 12 hours and spontaneously reverted to normal 9 days later.
\end{abstract}

Conclusion: In patients with large ventricular septal defects, large atrial septal occluders may be used. In cases with risk of pulmonary vascular disease, a safer option would be to close the defect using a manually fenestrated device.

Keywords: Transcatheter closure, Home-made fenestration, Muscular ventricular septal defect

\section{Background}

Hemodynamically significant muscular ventricular septal defects (VSD) in children after the infantile period are a rare occurrence and ideal for transcatheter closure [1]. Depending on the type, size, location and number of defects, it may be necessary to use an off-label device or multiple

\footnotetext{
*Correspondence: turkaysaritas@yahoo.com

'Department of Pediatric Cardiology, Istanbul Medipol University, Faculty of Medicine, Istanbul, Turkey

${ }^{3}$ Goztepe Mh. Dr. Rifat Pasa Sk. No: 21/33, 34730, Goztepe, Kadikoy, Istanbul, Turkey

Full list of author information is available at the end of the article
}

devices [2-4]. Additionally, in cases of severe concomitant pulmonary hypertension (PHT), it may be necessary to fenestrate the device [5]. In this report, we present an 18-year old patient with a large mid-trabecular VSD and severe PHT that underwent percutaneous closure of the defect with a home-made fenestrated atrial septal occluder (ASO).

\section{Case presentation}

An 18-year-old female patient referred to us with complaints of dyspnea (NYHA score of 2-3). An apical rumble and a harsh second heart sound were heard in physical examination. Echocardiographic examination revealed a 


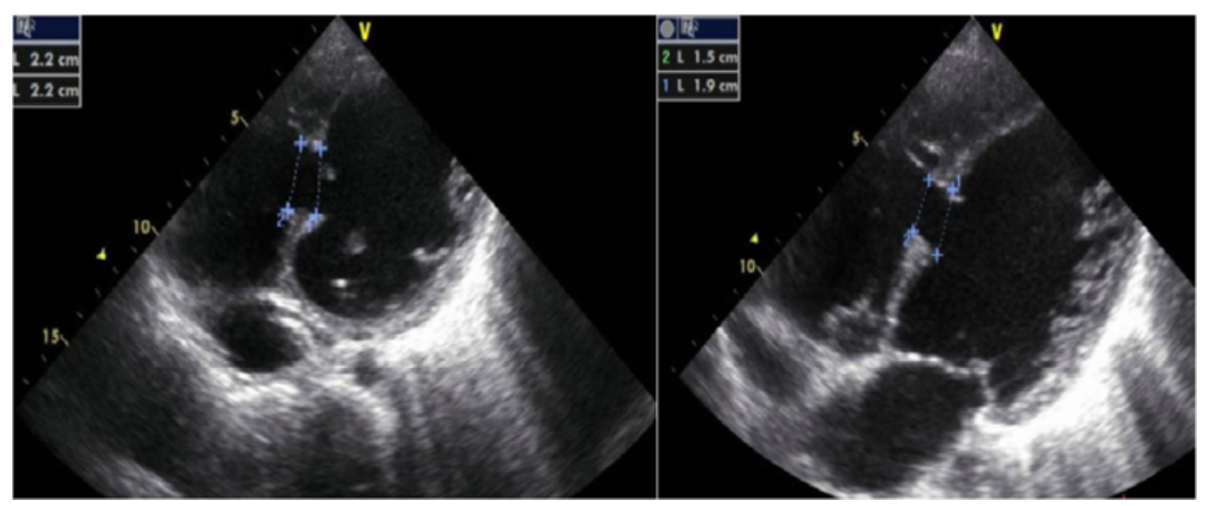

Figure 1 Diameters of VSD on transthoracic echocardiogram.

large mid-trabecular VSD with bidirectional shunt and the widest diameter measuring $22 \mathrm{~mm}$ on 2D echocardiography (ECHO) (Figure 1; Additional file 1). The defect was located $9 \mathrm{~mm}$ proximal to the moderator band. Left and right heart cavities were enlarged; left ventricle, left atrium and right ventricle end-diastolic diameters were respectively $68 \mathrm{~mm}(Z$ score: +8.23$), 44 \mathrm{~mm}(\mathrm{Z}$ score: +6.22$)$ and $41 \mathrm{~mm}(\mathrm{Z}$ score: +10.3$)$. Pulmonary artery diastolic pressure, which we were able to measure due to pulmonary insufficiency, was $58 \mathrm{mmHg}$. Before and after the vasoreactivity test with inhaled iloprost performed during cardiac catheterization, average aortic pressure was $65 \rightarrow 86 \mathrm{mmHg}$, average pulmonary artery pressure: $58 \rightarrow 73 \mathrm{mmHg}$, Qp/Qs: $1.6 \rightarrow 3.2$, PVR: $4.6 \rightarrow$ $4.3 \mathrm{Wood} / \mathrm{U} / \mathrm{m}^{2}$ and PVR/SVR: $0.5 \rightarrow 0.2$ (Table 1 ).

On left-ventricular angiocardiogram, the largest enddiastolic defect diameter was $21 \mathrm{~mm}$. The closure procedure was performed with transthoracic echocardiographic guidance, using a $24 \mathrm{~mm}$ Cera septal occluder (CSO, Lifetech Scientific Co., ltd, Shenzhen, China) and a 14 F sheath dilator to make a 4.5-5 mm opening (Figures 2 and 3). Measured immediately after the procedure and during cardiac catheterization one month later, average aortic pressure was $75 \rightarrow 75 \mathrm{mmHg}$, average pulmonary artery pressure: $66 \rightarrow 30 \mathrm{mmHg}$, Qp/Qs $1.5 \rightarrow 1.4$, PVR: $4.4 \rightarrow$ $2.9 \mathrm{Wood} / \mathrm{U} / \mathrm{m}^{2}$ and PVR/SVR: $0.4 \rightarrow 0.2$ (Table 1 ).

Transthoracic echocardiographic examination performed 24 hours after the procedure showed a max 35-40 $\mathrm{mmHg}$ gradient between the left and right ventricles through the fenestration. At the follow-up examination one month later, the gradient level was the same and there was a 50 drop in pulmonary artery diastolic pressure compared to the preprocedure value.

The patient had a normal sinus rhythm prior to the procedure. After the procedure, we observed sporadic early ventricular systoles and a nodal rhythm disorder that started after approximately 12 hours and spontaneously reverted to normal 9 days later. The patient was put on dexamethasone for the nodal rhythm. Propranolol was started after Holter ECG showed intermittent non-sustained ventricular tachycardia (VT) attacks. When the initially intermittent nodal rhythm became permanent, the patient was switched to sotalol. The last ECG and Holter ECG showed a normal sinus rhythm with sporadic early ventricular systoles and no VT attacks (Figures 4 and 5). Holter ECG performed 1 month later showed no early ventricular systoles.

The patient was closely monitored for hemolysis due to the fenestration of the device, but none was observed.

The patient's dyspnea (NYHA score 2-3 upon admission) started to recede on the $4-5^{\text {th }}$ day after the procedure and was graded as 1-2 in the follow-up examination 1 month later.

\section{Discussion}

Transcatheter closure of congenital and acquired VSDs is a procedure that has gained widespread use in the recent

Table 1 Hemodynamic parameters during the vasoreactivity test, immediately after the procedure and one month later

\begin{tabular}{lcccc}
\hline & Before vasoreactivity test & After vasoreactivity test & Immediately after closure & One month later \\
\hline Mean systemic pressure $(\mathbf{m m H g})$ & 65 & 86 & 75 & 75 \\
Mean PA pressure $(\mathbf{m m H g})$ & 58 & 73 & 66 & 30 \\
Qp/Qs & 1.6 & 3.2 & 1.5 & 1.4 \\
PVR (WU/m ${ }^{2}$ ) & 4.6 & 4.3 & 4.4 & 2.9 \\
PVR/SVR & 0.5 & 0.2 & 0.4 & 0.2 \\
\hline
\end{tabular}

PA: Pulmonary artery; Qp/Qs: Pulmonary to systemic flow ratio; PVR: Pulmonary vascular resistance; WU: Wood Unit; PVR/SVR: Pulmonary vascular resistance to systemic vascular resistance ratio. 


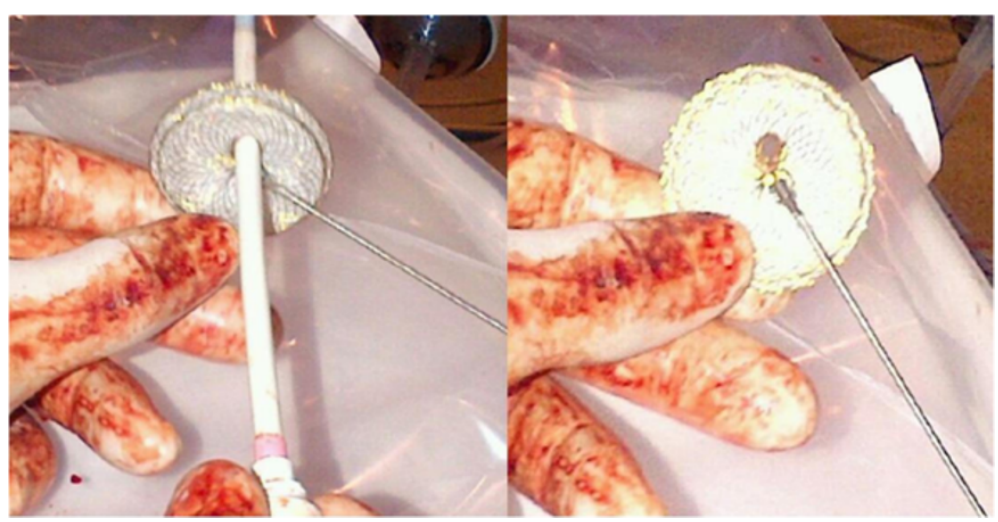

Figure 2 Fenestration procedure and the fenestrated ASD device.

years. Initially, VSD closure was performed using devices designed for atrial septal defects (ASD), but eventually special devices intended for closure of muscular and perimembranous defects were developed [6,7]. Although there has been remarkable progress in production of specifically designed occlusion devices, demand for off-label or custommade devices suitable for various types of defects remains high $[2,3,8]$.

Transcatheter closure of postinfarction, traumatic or residual postoperative VSDs is mainly performed using ASOs $[3,9]$. As ECHO and angiocardiographic measurements showed a large defect size in our patient, we elected to use a $24 \mathrm{~mm}$ ASO, a device 2-3 $\mathrm{mm}$ larger than the defect. The defect could have been closed using a $24 \mathrm{~mm}$ muscular VSD occluder or a post-myocardial infarction muscular VSD occluder as well; however, we preferred the ASO as it would allow for more convenient manual fenestration.

Long-term pulmonary hypertension may lead to a fixed increase in pulmonary vascular resistance. In some patients that have undergone VSD closure, pulmonary hypertension may persist or regress very slowly, in which case there may

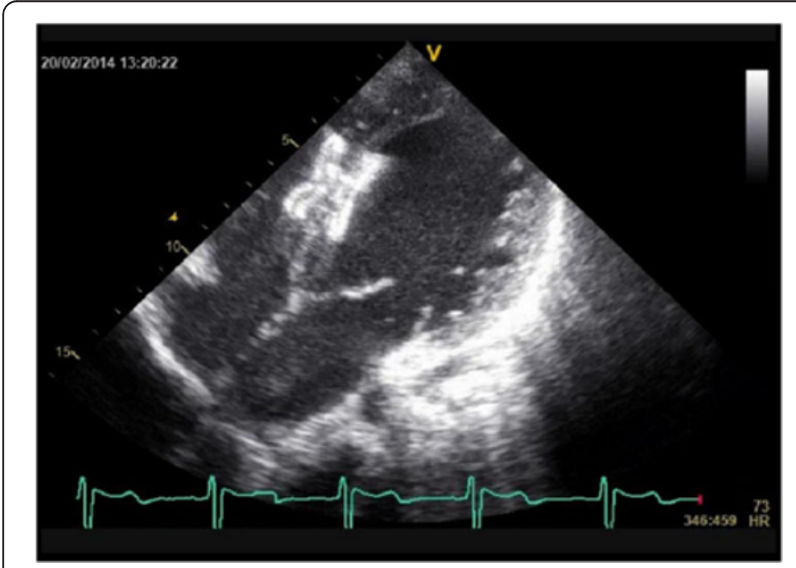

Figure $32 \mathrm{D}$ imaging of device after the procedure. be hypertensive crisis-like exacerbations requiring long-term ventilation and specific treatment. In VSD cases with high pulmonary resistance, post-operative pulmonary hypertensive crises as well as acute congestive heart failure and respiratory failure may prove fatal. For these reasons, surgeons nowadays are instructed to leave an opening in the VSD patch or to use a flap patch that allows for left-to-right shunt [10]. For the same reasons, fenestration is now performed on transcatheter occluder devices. Home-made fenestrated devices have been safely used in cases of ASD with severe PHT under the assumption that fenestrated occluders reduce left-to-right shunt and in the long run, lower pulmonary arterial pressure [5]. Bruch et al. reported using a homemade fenestrated ASO that allowed for a minor to moderate bidirectional shunt in elderly patients with $\mathrm{PHT}$ and/or right heart failure. While the authors did not see any deterioration of right or left heart failure in their patient group, they observed an impressive symptomatic benefit [11].

As our patient was an 18-year-old with a large VSD and severe PHT, we performed a vasoreactivity test in order to assess whether the defect could be closed. The patient's left heart cavities were found to be enlarged, the pulmonary artery flow velocity was high and the response to the vasoreactivity test was positive, so the patient was deemed a suitable candidate for transcatheter closure. However, as the patient was a young adult with bidirectional shunt and an enlarged right ventricle and pulmonary artery, we debated whether or not to fenestrate the device.

A review of literature did not reveal any cases with manually fenestrated devices used for closure of VSDs in patients with severe PHT. However, based on the Bruch study mentioned above and others that used fenestrated ASOs, we assumed that such a device in our case would allow for bidirectional shunt and could possibly reduce PHT in the long run. While no satisfactory reduction in pulmonary artery pressure was seen immediately after the procedure, transthoracic ECHO performed 24 hours later showed leftto-right gradient of $35-40 \mathrm{mmHg}$ through the fenestration. 


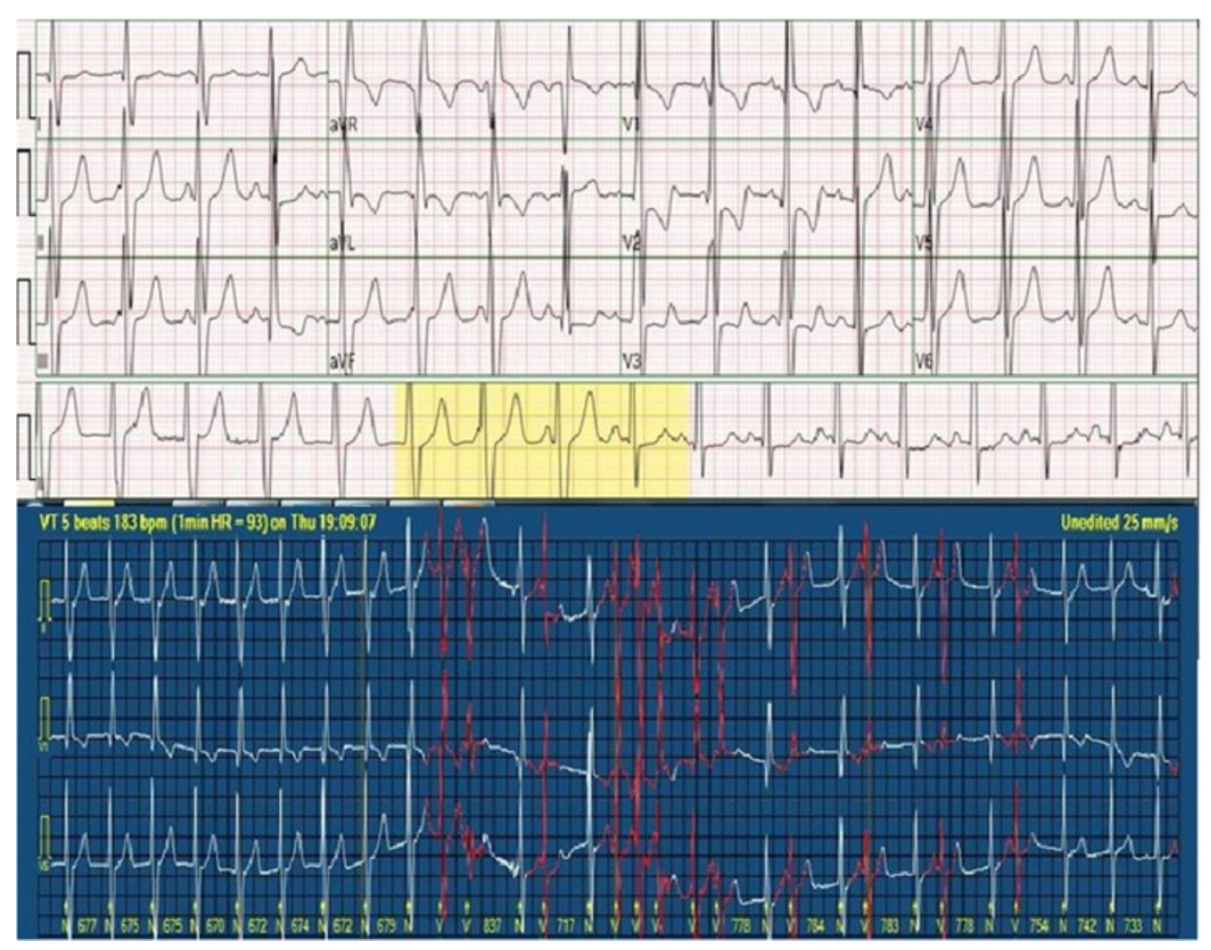

Figure 4 Nodal rhythm (top strip) on ECG and non-sustained VT attack (bottom strip) on 24-hour Holter ECG.

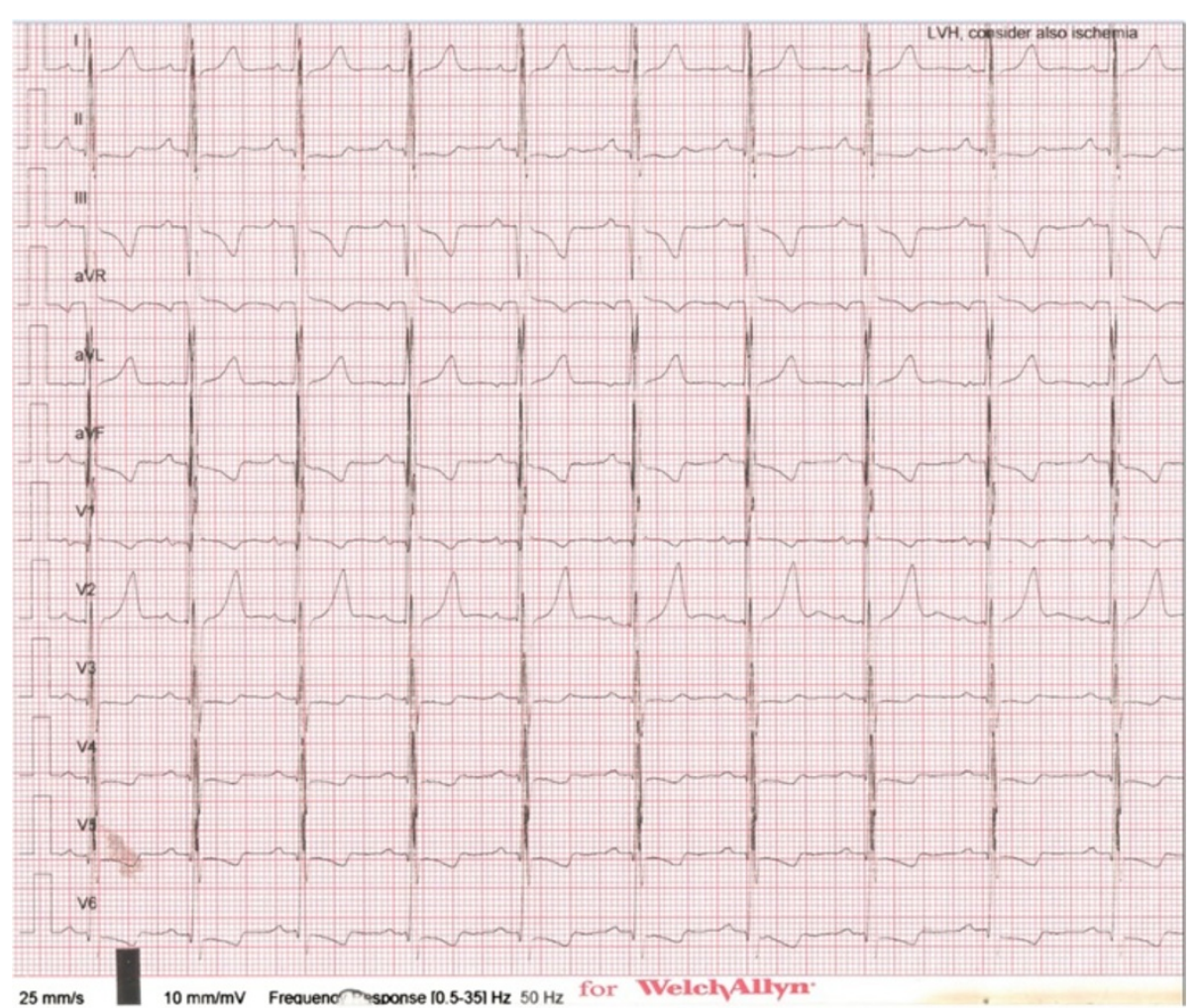

Figure 5 Patient ECG on day 9. 
In the follow-up examination 1 month later, the gradient value was the same, average aortic pressure was $75 \mathrm{mmHg}$, average pulmonary artery pressure was $30 \mathrm{mmHg}$ and PVR/SVR was 0.2.

Both minor and major complications such as hemolysis, cardiac perforation, device malposition, thromboembolism, temporary or permanent rhythm disorders, endocarditis and death may occur during, immediately after or in the follow-up of transcatheter defect closure $[4,12]$. In our case, a nodal rhythm started 12 hours after the procedure and spontaneously reverted to normal 9 days later. Temporary junctional rhythms following closure of muscular VSDs have also been reported in the literature $[12,13]$.

Heart block occurring after placement of an oversized device can be attributed to the expansion of the device against the conducting tissue. Even if the device is not oversized, it may cause an inflammatory reaction or formation of scarring in the conduction tissue, in which case steroid therapy may be useful [14]. The nodal rhythm in our patient can be attributed to the fact that an ASO has a larger left disc and a narrower gap between the discs than a VSD device, which may have put pressure on the conduction system or the neighboring tissues.

\section{Conclusion}

In patients with large VSDs, large ASDs may be used as well as large muscular VSD occluders or post-myocardial infarction muscular VSD occluders. In cases with risk of pulmonary vascular disease, a safer option would be to close the defect using a manually fenestrated device.

\section{Consent}

Written informed consent was obtained from the patient for publication of this case report and any accompanying images. A copy of the written consent is available for review by the Editor of this journal.

\section{Additional file}

Additional file 1: 2D and color flow echocardiographic imaging of VSD.

\section{Abbreviations}

VSD: Ventricular septal defects; PHT: Pulmonary hypertension; ASO: Atrial septal occluder; ECHO: Echocardiography; VT: Ventricular tachycardia; ASD: Atrial septal defects.

\section{Competing interests}

The authors declare that they have no financial and/or non-financial competing interests.

\section{Authors' contributions}

AE and TS performed the transcatheter intervention. HK gathered patient data. CA and VT treated the patient's arrhythmia, and contributed to the conception of the report. TS wrote the report. AE checked the report in terms of English grammar. All authors gave their final acceptance to the submission of this report. All authors read and approved the final manuscript.

\section{Acknowledgements}

We thank Layla Rahimov who provided medical writing of this report in English.

\section{Author details}

${ }^{1}$ Department of Pediatric Cardiology, Istanbul Medipol University, Faculty of Medicine, Istanbul, Turkey. ${ }^{2}$ Department of Pediatric and Genetic Arrhythmia, Istanbul Medipol University, Faculty of Medicine, Istanbul, Turkey. ${ }^{3}$ Goztepe Mh. Dr. Rifat Pasa Sk. No: 21/33, 34730, Goztepe, Kadikoy, Istanbul, Turkey.

Received: 23 March 2014 Accepted: 30 May 2014

Published: 9 June 2014

\section{References}

1. Arora R, Trehan V, Thakur AK, Mehta V, Sengupta PP, Nigam M: Transcatheter closure of congenital muscular ventricular septal defect. J Interv Cardiol 2004, 17:109-115.

2. Baspinar O, Irdem A, Kilinc M: Off-label use of Amplatzer devices in congenital heart disorders during childhood. Acta Cardiol 2013, 68:31-35.

3. Marinakis A, Vydt T, Dens J, Gewillig M, Van Deyk K, Budts W: Percutaneous transcatheter ventricular septal defect closure in adults with Amplatzer septal occluders. Acta Cardiol 2007, 62:391-395.

4. Holzer R, Balzer D, Cao QL, Lock K, Hijazi ZM: Device closure of muscular ventricular septal defects using the Amplatzer muscular ventricular septal defect occluder: immediate and mid-term results of a U.S. registry. J Am Coll Cardiol 2004, 43:1257-1263.

5. Dell'avvocata F, Rigatelli G, Cardaioli P, Giordan M: Home-made fenestrated amplatzer occluder for atrial septal defect and pulmonary arterial hypertension. J Geriatr Cardiol 2011, 8:127-129.

6. Michel-Behnke I, Le TP, Waldecker B, Akintuerk H, Valeske K, Schranz D: Percutaneous closure of congenital and acquired ventricular septal defects-considerations on selection of the occlusion device. J Interv Cardiol 2005, 18:89-99.

7. Fu YC: Transcatheter device closure of muscular ventricular septal defect. Pediatr Neonatol 2011, 52:3-4.

8. Rohit MK, Gupta A: Transcatheter closure of large patent ductus arteriosus using custom made devices. Catheter Cardiovasc Interv 2014, 00:00-00.

9. Suh WM, Kern MJ: Transcatheter closure of a traumatic VSD in an adult requiring an ASD occluder device. Catheter Cardiovasc Interv 2009, 74:1120-1125

10. Rao PS, Raju V, Narayana M: Flap valved closure of ventricular septal defects with increased pulmonary vascular resistance. Interact Cardiovasc Thorac Surg 2010, 11:577-580.

11. Bruch L, Winkelmann A, Sonntag S, Scherf F, Rux S, Grad MO, Kleber FX: Fenestrated occluders for treatment of ASD in elderly patients with pulmonary hypertension and/or right heart failure. J Interv Cardiol 2008, 21:44-49.

12. Chessa M, Carminati M, Cao QL, Butera G, Giusti S, Bini RM, Hijazi ZM: Transcatheter closure of congenital and acquired muscular ventricular septal defects using the Amplatzer device. J Invasive Cardiol 2002, 14:322-327.

13. Hijazi ZM, Hakim F, Al-Fadley F, Abdelhamid J, Cao QL: Transcatheter closure of single muscular ventricular septal defects using the amplatzer muscular VSD occluder: initial results and technical considerations. Catheter Cardiovasc Interv 2000, 49:167-172.

14. Butera G, Chessa M, Carminati M: Percutaneous closure of ventricular septal defects. Cardiol Young 2007, 17:243-253.

doi:10.1186/1471-2261-14-74

Cite this article as: Kamali et al:: Percutaneous closure of large VSD using a home-made fenestrated atrial septal occluder in 18-year-old with pulmonary hypertension. BMC Cardiovascular Disorders 2014 14:74. 